\title{
Design and Simulation of Temperature Sensor based on Long Period Grating in Liquid Filled Photonic Crystal Fiber
}

\author{
R. Boufenar ${ }^{1,2}$, M. Bouamar ${ }^{1}$ and A. Hocini ${ }^{1}$ \\ ${ }^{1}$ Laboratoire d'Analyse des Signaux et Systemes, Department of Electronics, Mohamed Boudiaf University, \\ M'sila 28000, Algeria \\ ${ }^{2}$ Nuclear research center, BP 180 Ain Oussera/Djelfa 17000/Algeria. \\ *Corresponding author: Boufenar Rabah \\ E-mail:rabouf@yahoo.fr
}

\begin{abstract}
In this paper a novel measurement method for temperature has been proposed and investigated based on an ethanol-filled photonic crystal fiber long period grating (PCF-LPG). The ethanol-filled PCF-LPG is sensitive to the refractive index (RI) of internal filled liquid, which will produce a large RI change with surrounding temperature variation and affect the coupling between the core mode and the forward propagating cladding modes. The results indicated that the sensitivity of the proposed temperature sensor can reach $9 \mathrm{~nm} /{ }^{\circ} \mathrm{C}$ in the range of $20^{\circ} \mathrm{C}$ to $80{ }^{\circ} \mathrm{C}$ which is 90 times higher than that of conventional LPG temperature sensors. The simplicity and the excellent performance of our proposed device make it potential for highprecision temperature measurement.
\end{abstract}

\section{Introduction}

Fiber optic based sensors are rapidly gaining popularity due to their many advantages over conventional techniques, such as immunity to electromagnetic interference, remote sensing, and ease in handling, low cost and small size [1].

Fiber Bragg grating (FBG) is one of the most important photonic devices in fiber optic communication and sensing [2]. It is fabricated by forming a periodic refractive-index modulation along the fiber. When light is guided along the core of the FBG, it gets reflected by successive grating planes; The contributions of reflected light from different grating planes add constructively for a particular wavelength.

The interrogation of a FBG sensor can be realized simply by utilizing this wavelength dependent transmission loss.

Long period fiber Bragg gratings, often called long period gratings (LPG) [3], have been known for over two decades, is an axially periodic refractive index variation inscribed in the core of an optical fiber, it has a period in the hundreds of micrometers, and couples the guided light inside the fiber core into the cladding modes at certain discrete wavelengths (also known as resonance wavelengths). There are some dips in the transmission spectrum at wavelengths that satisfy the resonant condition.

Fiber-optic long period gratings (LPG) have received much attention in recent years due to their applications in optical fiber communications and sensing [4]. LPG devices have been demonstrated for use as spectral shapers [5], mode converters [6] and temperature, strain, refractive index and bend sensors [7].

Since 1999, researchers have fabricated LPGs in a new class of fibers, known as photonic crystal fibers (PCFs) that emerged in recent years. Typically, these fibers incorporate a number of air holes that run along the length of the fiber and have a variety of different geometries and sizes [8,9].

Photonic crystal fibers (PCFs) have attracted great research interest owing to their wide range of unique optical properties that cannot be realized by using conventional single mode fiber, such as endless single mode guiding, nonlinearity, tailorable chromatic dispersion, and high birefringence.

Fiber long period gratings based on PCFs (PCF-LPG) are novel optical devices. They utilize periodic refractive index and geometry perturbations along the length of a PCF.

When the period of the perturbation matches the beat length between the fundamental core mode and the co-propagating cladding mode, or between two cladding modes, the perturbation facilitates coupling between the two modes [10].

Many researchers are interested in the properties and the applications of fiber long period gratings based on PCFs.

In the field of temperature sensor much research has been conducted in recent years [11]. Qian et al. [12] reported a compact temperature sensor based on a fiber loop mirror (FLM) combined with an alcohol-filled high birefringence photonic crystal fiber, and experimental results demonstrated that the temperature sensitivity can reach up to $6.6 \mathrm{~nm} /{ }^{\circ} \mathrm{C}$, but the temperature detecting window was just $14^{\circ} \mathrm{C}$. Di Wu et al. [13] proposed a compact temperature sensor based on FLM with a 
short length of ethanol-filled highly birefringent photonic crystal fiber (HB-PCF) inserted as a temperature element. The characteristics and temperature response of the sensor are theoretically and experimentally investigated.

Due to the high temperature sensitivity of the filled ethanol, the temperature response of the ethanol-filled HB-PCF FLM presents a temperature sensitivity of $0.8833 \mathrm{~nm} /{ }^{\circ} \mathrm{C}$.

Wenwen Qian et al. [14] reported a compact temperature sensor based on a modal interferometer made of a short ethanolfilled photonic crystal fiber combined with a fully collapsed splicing with single-mode fibers (SMFs) and the temperature sensitivity as high as $0.35 \mathrm{~nm} /{ }^{\circ} \mathrm{C}$ was obtained, where the temperature changed from $20^{\circ} \mathrm{C}$ to $50^{\circ} \mathrm{C}$.

The disadvantage of these infiltrated PCF temperature sensors is that the fabrication process is complicated and expensive, also due to complicated sensing structure, the loop structure measurement systems always show poor stability [15, $16]$.

The material with a high thermo-optic coefficient will produce a large refractive index (RI) change with surrounding temperature variation, so the measurement of temperature can be achieved by monitoring the RI change. LPG written in PCF can couple the core mode to the forward propagating cladding modes, and shows relatively high RI resolution because of the strong interaction between cladding modes and filled medium [17], so it can be used for the measurement of temperature.

Ethanol is a transparent liquid with a high thermo-optic coefficient and its refractive index is lower than that of the silica core region, which is easy to be integrated with PCF.

In this paper, we propose a high sensitivity temperature sensor based on photonic crystal fiber long period grating filled with ethanol and we report a numerical analysis of modal coupling properties and resonance wavelength of (PCF- LPG) in response to changes in refractive indices of the medium contained in the cladding air channels.

A full vectorial mode solver based on finite element method and absorbing perfectly matched layer boundary condition (PML) is used for the analysis.

The response of the LPG based PCF to temperature was studied and the sensitivity was investigated by monitoring the shifts of the dip wavelength.

\section{Working Principle}

Long-period fiber Bragg grating (LPG) is a process to periodically change the core cladding difference in refractive index along the length of an optical fiber.

Up to date, various ways have been reported for creating localized refractive index changes along an optical fiber, including UV light [18], electric arc discharge [19], focused infrared femtosecond laser pulses [20] and $\mathrm{CO}_{2}$ laser [21].

Mode coupling happens when phase matching condition between the fundamental core mode and a particular cladding mode is satisfied. The phase matching condition required for coupling is given by [22]:

$$
\lambda_{\text {res }}=\left(n_{c o} \text { eff }-n_{c l, m}{ }^{e f f}\right) \cdot \Lambda_{G}
$$

In which $n_{c o}{ }^{e f f}$ and $n_{c l, m}{ }^{e f f}$ are the effective indices of the guided core mode and the $m$ cladding mode, respectively; $\lambda_{\text {res }}$ is the center wavelength of the transmission resonance; and $\Lambda_{G}$ is the period of refractive index modulation.

The light traveling in core modes experiences a high loss, which gives attenuation bands at resonance wavelengths in the transmission spectrum.

As temperature changes, effective indices of the core mode and the cladding mode change obviously because of the high temperature sensitivity of the liquid filled (ethanol) so that the difference of effective indices of the core mode and the cladding mode is highly affected by temperature. Therefore, the resonant wavelength which arises from light coupling from the fundamental mode to the forward propagating cladding modes is very sensitive to temperature due to the mode couplings. By monitoring the output resonant wavelength, we can realize the detection of temperature change.

\section{Structure Design and Numerical Calculation}

The geometrical structure of the proposed sensor as shown in Figure 1 and Figure 2, consists of a pure silica core with a diameter of $8.80 \mu \mathrm{m}$, which is surrounded by six ring layers of air holes in a silica matrix, arranged in a regular hexagonal pattern; the diameter of the air holes is $2.20 \mu \mathrm{m}$, while the pitch and the period grating $\left(\Lambda_{G}\right)$ are $5.30 \mu \mathrm{m}$ and $650 \mu \mathrm{m}$ respectively. The index modulation is $1 \times 10^{-4}$ and the gratings length is $6.5 \mathrm{~mm}$.

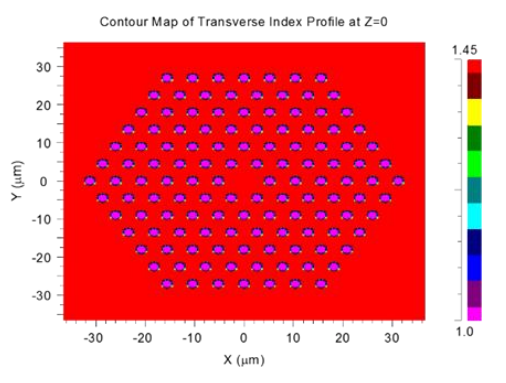

Figure 1: Cross section of the proposed (PCF-LPG) sensor.

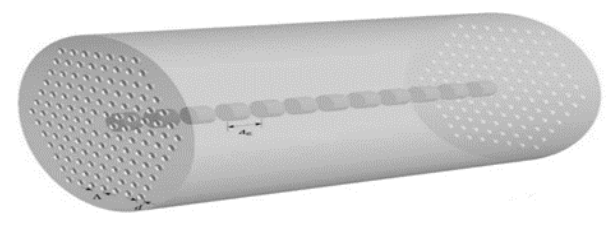

Figure 2: Schematic representation of the proposed PCF-LPG Temperature sensor. 
The cladding holes of the PCF are filled with ethanol. Whose refractive index strongly depends on temperature according to the relation [23]:

$$
n=n_{0}-\alpha\left(T-T_{0}\right)
$$

Where $n$ and $n_{0}$ are the refractive indices at temperature $T$ and $T_{0}$ respectively.

The refractive index of the silica fibers is set as a constant at all temperatures during the simulations because the thermooptic coefficient $\alpha$ of liquid ethanol is: $3.94 \times 10^{-4} / K$ (which is two orders of magnitude higher than that of pure silica $\alpha=$ $\left.8.6 \cdot 10^{-6} / K\right)$. Finally, $n_{0}$ is initialized to be 1.36048 at the input wavelength and $T_{0}=20^{\circ} \mathrm{C}$.

The phase-matching condition required for coupling is given by [22]:

$$
\lambda_{\text {res }}=\left(n_{c o}{ }^{e f f}-n_{c l, m}{ }^{e f f}\right) \cdot \Lambda_{G}
$$

Where: $n_{c o}$ eff is the effective index of the guided core mode and $n_{c l, m}$ eff the $m$ cladding mode; $\lambda_{\text {res }}$ is the center wavelength of the transmission resonance; and $\Lambda_{G}$ is the period of refractive index modulation.

In this work, we have employed an efficient full vector finite element method (FV-FEM) with anisotropic perfectly matched layers (PML) for accurate modeling of PCF-LPG based temperature sensor. Commercially available Rsoft $\AA$ software is used to achieve these calculations.

Due to symmetry reasons, it is sufficient to calculate only one quarter of the PCF structure.

\section{Analysis Method}

Among the full-vectorial methods used in modeling PCFs, the finite element method (FEM) [24] is particularly effective for handling curved interfaces with high accuracy, and it is obviously a good choice for the analysis of combined circular and elliptical shape.

In the modal solution approach based on the FV-FEM, the intricate cross section of the PCF can be accurately represented using many triangles of different shapes and sizes. This flexibility makes the FV-FEM preferable to other approaches.

In this study, we have adopted an efficient FV-FEM with PMLs to predict all the propagation characteristics of the waveguide with high accuracy.

The fiber cross section representation is very accurate as the domain is divided into sub domains with triangular or quadrilateral shape, where any refractive index profiles can be properly represented.

Applying the variational FV-FEM procedure to Maxwell's equations, the following vector wave equation is derived [25].

$$
\nabla \times\left([s]^{-1} \nabla \times \vec{E}\right)-k_{0}{ }^{2} n^{2}[s] \vec{E}=0
$$

Where $k_{0}=2 \pi / \lambda$ the free-space wavenumber, $\lambda$ is the wavelength, $\vec{E}$ denotes the electric field, $n$ is the refractive index, $[s]$ is the PML matrix, and $[s]^{-1}$ is the inverse of the $[s]$ matrix.

When applying FV-FEM to PCFs, a curvilinear hybrid edge/nodal element [26] is very useful for avoiding spurious solutions and for accurately modeling curved boundaries of air holes.

Dividing the fiber cross section into a number of the curvilinear hybrid elements, we can obtain from Eq. (4) the following eigenvalue equations:

$$
[k]\{E\}=k_{0}{ }^{2} n_{e f f}{ }^{2}[M]\{E\}
$$

Where $[k]$ and $[M]$ are the finite element matrices, $\{E\}$ is the discretized electric field vector consisting of the edge and nodal variables, and $n_{\text {eff }}$ is the effective index.

Moreover, in order to model infinite PCF with twodimensional finite-geometry (i.e., to enclose the computational domain without affecting the numerical solution), it is necessary to use anisotropic perfectly matched layers (PMLs) which are placed in the contact with the outermost boundary.

\section{Results and Discussion}

In this work, we have employed the full vector finite element method (FV- FEM) with anisotropic perfectly matched layers (PMLs), which is highly suitable for the analysis of such structure. Commercially available Rsoft ${ }^{\circledR}$ software is used to achieve these calculations.

To investigate the temperature effect on the proposed ethanol filled PCF-LPG based temperature sensor, different temperatures: $20^{\circ} \mathrm{C} ; 30^{\circ} \mathrm{C} ; 40{ }^{\circ} \mathrm{C} ; 50{ }^{\circ} \mathrm{C} ; 60^{\circ} \mathrm{C} ; 70{ }^{\circ} \mathrm{C}$; and 80 ${ }^{\circ} \mathrm{C}$ have been defined.

The refractive index of the infiltrated liquid at initial temperature $T_{0}=20^{\circ} \mathrm{C}$ is set to be: $n_{0}=1.36048$.

Figure 3 shows the transmission spectrum of the proposed PCF taken after filling the liquid ethanol.

As shown in Figure 3, the transmission spectrum for such structure presents two notches. One located at the wavelength $1.32 \mu \mathrm{m}$ and the other at the wavelength $1.55 \mu \mathrm{m}$, which are due to the fundamental core mode coupling to two different cladding modes.

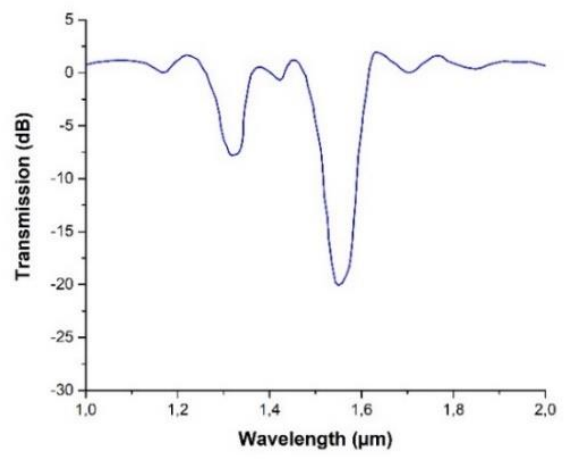

Figure 3: Transmission spectrum of the proposed PCF-LPG. 
Figure 4 (a) and (b) shows the intensity distribution of the core and the cladding mode respectively. Which are considered as the two coupling modes in our PCF-LPG.

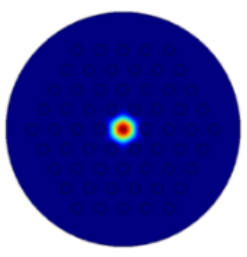

(a)

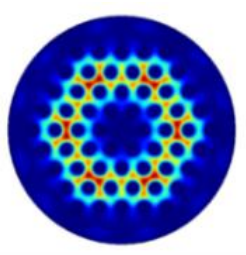

(b)
Figure 4: Intensity distribution of the core and the cladding mode.

The dip at the wavelength $1,55 \mu \mathrm{m}$ is nearly $-20 \mathrm{~dB}$. By monitoring this resonant wavelength, we can realize the detection of temperature change.

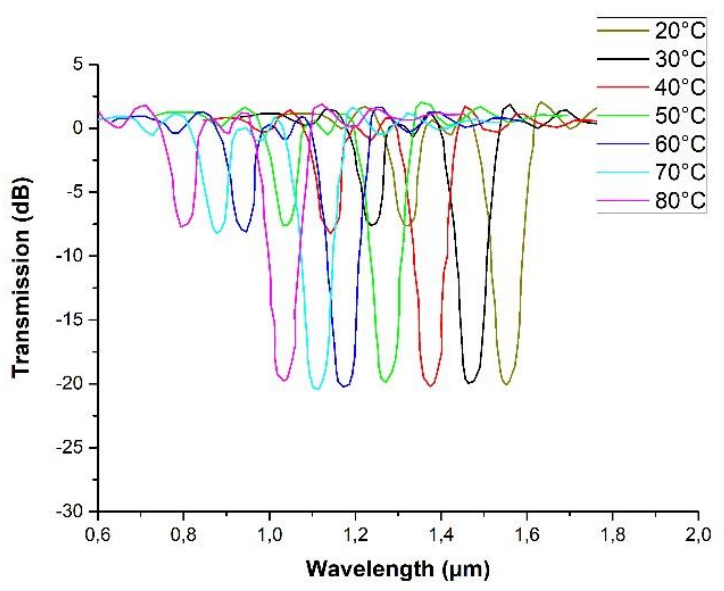

Figure 5: Resonant wavelength of the proposed ethanol-filled PCF-LPG for various temperatures.

As shown in Figure 5, the resonant wavelength of the proposed ethanol filled PCF-LPG shift to a shorter wavelength when temperature increases.

That is because ethanol exhibits a highly negative thermooptic coefficient : $-3.94 \times 10^{-4} / K$, therefore as temperature rising, effective indices of the core mode and the cladding mode decrease obviously since the refractive index of the infiltrated liquid decreases linearly with temperature, induces a blue shifting of the dip wavelength.

Simulation results showed that the resonant wavelength blue-shifted by $545 \mathrm{~nm}$ as temperature changed from $20^{\circ} \mathrm{C}$ to $80^{\circ} \mathrm{C}$.

As shown in Figure 6, the function curve of the resonant wavelength of ethanol filled PCF-LPG shifts downwards when temperature increases, and is linearly proportional with temperature.

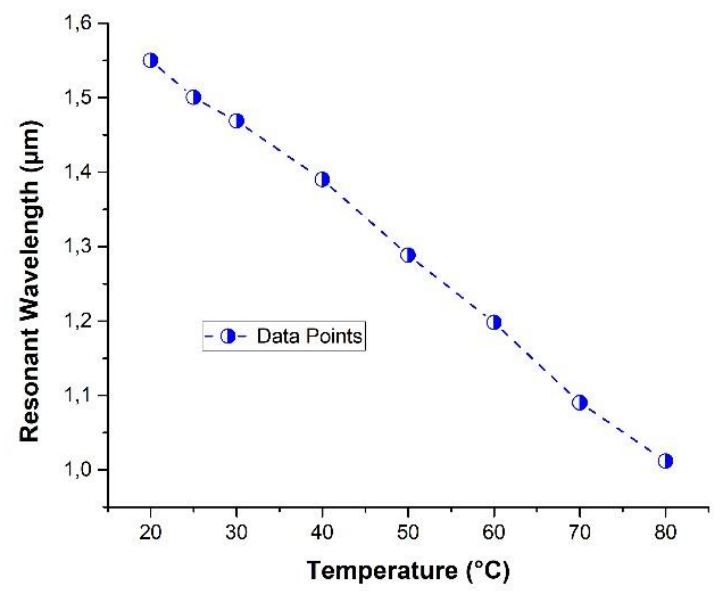

Figure 6: Resonant wavelength of ethanol-filled PCF-LPG as a function of temperature.

The relationship between the resonant wavelength and temperature can be fitted as:

$$
\lambda_{\text {res }}(n m)=-9.0794 T+1738.7
$$

When the gratting period $\Lambda_{G}$ of the ethanol filled PCF-LPG is $650 \mu \mathrm{m}$, and the fitting degree is as high as 0.9985 , as shown in Figure 7.

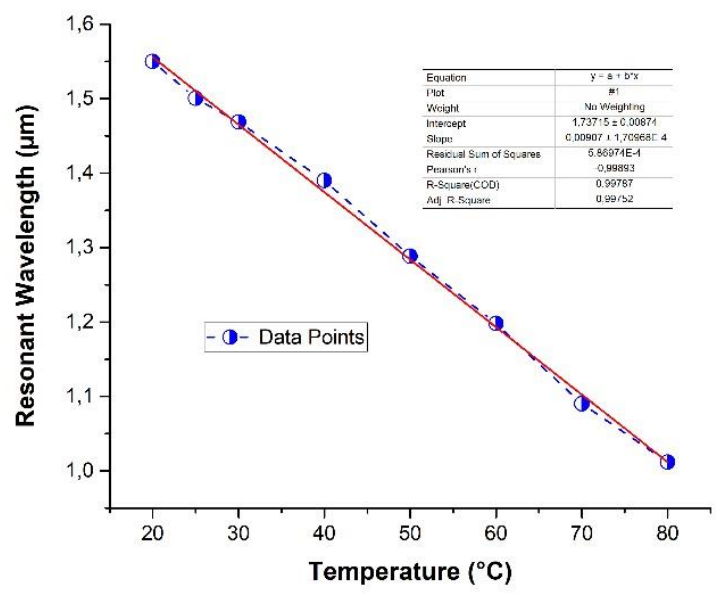

Figure 7: Resonant wavelength versus temperature after linear fitting of simulation data.

The sensitivity $\left(S=\Delta \lambda_{\text {res }} /_{\Delta T}\right) \quad$ is a key performance indicator. It is calculated as the ratio of the resonant wavelength shift and the change in temperature, it depends on the resonant wavelength shift with respect to the change of refractive index of the ethanol filled. The more shift is, the higher is the sensitivity. 
According to the numerical simulation, it can be seen that the sensitivity of this sensor is:

$$
S=\Delta \lambda_{\text {res }} /_{\Delta T}=9 \mathrm{~nm} /{ }^{\circ} \mathrm{C}
$$

For $20^{\circ} \mathrm{C}$ to $80^{\circ} \mathrm{C}$ detection range. Which is 900 times higher than that of unfilled PCF-LPG and 90 times higher than that of conventional LPG temperature sensor.

Thus, the proposed temperature sensor based on ethanolfilled PCF-LPG can detect temperature in a lager range with a linear relationship.

To investigate the effect of the LPG period on the resonnant wavelength and the temperature sensitivity, grating period is varied from $650 \mu \mathrm{m}$ to $900 \mu \mathrm{m}$, while other geometric parameters such air holes diametres and pitch is kept fixed.

Figure 8 shows the calculated resonance wavelength of the proposed ethanol filled PCF-LPG as a function of the grating period.

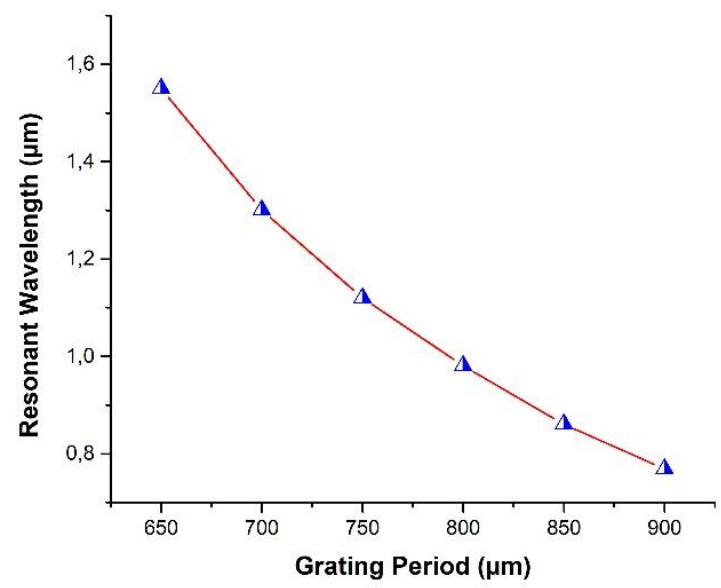

Figure 8: Resonant wavelength of ethanol-filled PCF-LPG as a function of the grating period.

As we show in Figure 8, the resonance wavelength of PCF-LPG filled with ethanol, decreases with the LPG period increasing. Figure 9 reviews the same basic trends, the temperaure sensitivity for the proposed ethanol filled PCF-LPG decreases when the grating period increases from $650 \mu \mathrm{m}$ to $900 \mu \mathrm{m}$.

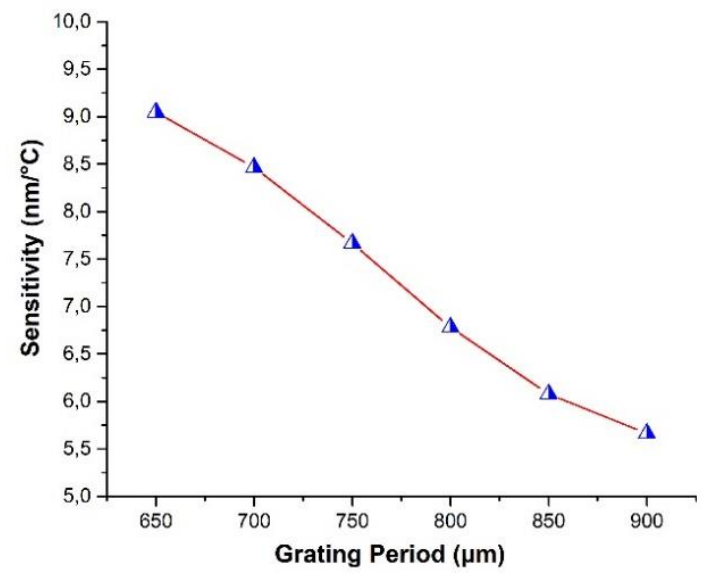

Figure 9: Temperaure sensitivity of ethanol-filled PCF-LPG as a function of the grating period.

In conclusion, we have shown theoretically that a properly designed of the ethanol filled LPG-PCF we can exhibit a robust sensitivity to the temperature.

Simulation results show that, the shorter the grating period, the longer the resonant wavelength, and the more sensitive PCFLPG is.

\section{Conclusion}

A highly sensitive temperature sensor based on ethanol-filled PCF-LPG has been proposed and simulated. The sensor can detect temperature in a very large range by monitoring the resonant wavelength of the ethanol filled PCF-LPG.

Simulation results show that the linearity of the resonant wavelength as a function of temperature keeps well, the sensor keeps good linearity in the measured temperature range.

the proposed sensor interrogation scheme can convert the temperature into resonant wavelength shift.

This system is simple and works accurately in environments in which the temperature can change in a wide range.

The sensitivity is as high as $9 \mathrm{~nm} /{ }^{\circ} \mathrm{C}$, that is 900 times higher than that of unfilled PCF-LPG and 90 times than that of conventional LPG temperature sensors.

Further enhancement of sensitivity is possible with improved PCF design.

\section{References}

[1] G. Rajan, “Optical Fiber Sensors: Advanced Techniques and Applications," CRC Press, February 12, 2015. 
[2] C.H. Tan, Y. G. Shee, B. K. Yap, F. R. Mahamd Adikan, "Fiber Bragg grating based sensing system: Early corrosion detection for structural health monitoring," Sensors and Actuators A: Physical, vol. 246, pp. 123-128., 2016.

[3] S. W. James, R. P. Tatam, "Optical fibre long-period grating sensors: Characteristics and application," Meas. Sci. Technol., vol. 14, R49-R61, 2003.

[4] A. Baliyan, S. Sital, U. Tiwari, R. Gupta, E. K. Sharma , "Long period fiber grating based sensor for the detection of triacylglycerides," Biosensors and Bioelectronics, vol.79, pp. 693-700, 2016.

[5] A. M. Vengsarkar, P. J. Lemaire, J. B. Judkins, V. Bhatia, T. Erdogan, J. E. Sipe, "Long period fiber gratings as band rejection filters," J. Lightwave Technol., vol.14, pp. 5864. 1996.

[6] S. Ramachandran, Z. Wang, M. Yan, "Bandwidth control of long-period grating based mode converters in few-mode fibers," Opt. Lett., vol.27, pp. 698-700, 2002.

[7] V. Bhatia, "Applications of long-period gratings to single and multi-parameter sensing," Opt. Express, vol. 4, pp. 457-466, 1999.

[8] P. Russell, "Photonic crystal fibers," Science, vol. 299(5605), pp. 358-362, 2003.

[9] J. Ju, W. Jin, "Long Period Gratings in Photonic Crystal Fibers," Photonic Sensors, vol. 2(1), pp. 65-70, 2012.

[10] K. Morishita, Y. Miyake, "Fabrication and resonance wavelengths of long-period gratings written in a pure silica photonic crystal fiber by the glass structure change," J. Lightwave Technol., vol. 22(2), pp. 625-630, 2004.

[11] L. Qian, L. Shuguang, C. Hailiang, Z. Fan, J. Li, "Photonic Crystal Fiber Temperature Sensor Based on Coupling Between Liquid-Core Mode and Defect Mode" IEEE Photonics Journal, vol. 7(2), 2015.

[12] W. Qian et al., "High-sensitivity temperature sensor based on an alcohol-filled photonic crystal fiber loop mirror," Opt. Lett., vol. 36, no. 9, pp. 1548-1550, May 2011.

[13] D. Wu, Y. Zhao, H. Hu, "Experimental research on FLM temperature sensor with an ethanol-filled photonic crystal fiber," Sensors and Actuators A, vol. 209, pp. 62-67, 2014.

[14] W. Qian, C.-L. Zhao, C. C. Chan, L. Hu, T. Li, W. C. Wong, P. Zu, X. Dong, "Temperature Sensing Based on Ethanol-Filled Photonic Crystal Fiber Modal Interferometer", IEEE Sensors Journal, vol.12(8), pp. $2593-2597,2012$.
[15] D. Leandro, M. Bravo, M. Lopez-Amo, "High resolution polarization-independent high birefringence fiber loop mirror sensor," Opt Express; vol.23(24):30985-90, 2015.

[16] C. Du, Q. Wang, Y. Zhao, J. Li, "Highly sensitive temperature sensor based on an isopropanol-filled photonic crystal fiber long period grating," Opt. Fiber Technol., vol., 34, pp.12-15, 2017.

[17] L. Rindorf, O. Bang, "Highly sensitive refractometer with a photonic-crystal fiber long-period grating," Opt. Lett., vol. 33(6), pp. 563-565, 2008.

[18] R. Z. V. Costa, R. C. Kamikawachi, M. Muller, J. L. Fabris, "Thermal characteristics of long-period gratings 266 nm UV-point-by-point induced," Opt. Commun., vol. 282(5), pp. 816-23, 2009.

[19] G. Rego, "Arc-Induced Long Period Fiber Gratings," journal of Sensors 2016, Article ID 3598634, 2016.

[20] F., Ahmed, H. E. Joe, B. K. Min, M. B. G. Ju., "Characterization of refractive index change and fabrication of long period gratings in pure silica fiber by femtosecond laser radiation," Opt. Laser Technol., vol. 74, pp. 119-124, 2015.

[21] J. M. P. Coelho, C. Silva, M. Nespereira, M. Abreu, `O. J. Reborda, "Writing of long period fiber gratings using $\mathrm{CO}_{2}$ laser radiation," in Advances in Optical Fiber Technology: Fundamental Optical Phenomena and Applications, chapter 9, pp. 287-314, In Tech, Rijeka, Croatia, 2015.

[22] J. Kanka, "Design of turn-around-point long-period gratings in a photonic crystal fiber for refractometry of gases," Sensors and Actuators B: Chemical, vol. 182, pp. 16-24, 2013.

[23] N. P. Barnes, M. S. Piltch, "temperature-dependent Sellmeier coefficient and coherent length for cadmium telluride," Opt. Soc. A., vol. 67(5), pp. 628-629, 1977.

[24] F. Brechet, J. Marcou, D. Pagnoux, P. Roy, “Complete Analysis of the characteristics of propagation into photonic crystal fibers by Finite Element Method," Opt. Fiber Technol., vol. 6, pp. 181-191, 2000.

[25] K. Saitoh, M. Koshiba, "Full-vectorial imaginary-distance beam propagation method based on a finite element scheme: application to photonic crystal fibers," IEEE J. Quantum Electron., vol. 38(7), pp. 927-933, 2002.

[26] M. Koshiba, Y. Tsuji, "Curvilinear hybrid edge/nodal elements with triangular shape for guided-wave problems," J. Lightwave Technol., vol. 18(5), pp. 737-743. 2000 . 